applicable recommendation would be to store separately in biobanks all human samples collected during the COVID-19 outbreak.

Biobanks can identify whether they have an appropriate quality-assurance system in place and demonstrate to end users that this system is being applied, together with standard security guarantees. The quality-assurance system also enables the transparent traceability of the samples requested from academia and from bioindustry. Traceability ultimately allows assessment of the quality of the samples and associated data. However, this comes with financial investment. The present crisis represents a new, critical and urgent challenge in the field of biobanking for cancer research.

\section{Paul Hofman ${ }^{1,2 凶}$, Pascal Puchois ${ }^{3}$,} Patrick Brest ${ }^{2}$, Hicham Lahlou ${ }^{3}$ and Daniel Simeon-Dubach ${ }^{4}$

${ }^{1}$ Université Côte d'Azur, CHU de Nice, Laboratory of Clinical and Experimental Pathology, Biobank (BB-0033-00025), FHU-OncoAge, Nice, France. ${ }^{2}$ Université Côte d'Azur, Institute of Research on Cancer and Ageing of Nice, CNRS, INSERM, Centre Antoine Lacassagne, FHU-OncoAge, Nice, France. ${ }^{3}$ Trans-Hit, Biomarkers, Montreal, Canada. ${ }^{4}$ Medservice, Biobanking Consulting \& Services, Walchwil, Switzerland.

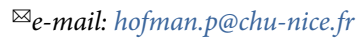

Published online: 7 May 2020

https://doi.org/10.1038/s41591-020-0890-8

$\square$

References

1. Wang, W. et al. J. Am. Med. Assoc. https://doi.org/10.1001/ jama.2020.3786 (2020)

2. Young, B. E. et al. J. Am. Med. Assoc. https://doi.org/10.1001/ jama.2020.3204 (2020).

3. Zhang, W. et al. Emerg. Microbes Infect. 9, 386-389 (2020).

4. Li, Y. C., Bai, W. Z. \& Hashikawa, T. J. Med. Virol. https://doi. org $/ 10.1002 / \mathrm{jmv} .25728$ (2020).

5. Diao, B. et al. medRxiv https://doi.org/10.1101/2020.03.04.20031120 (2020).

6. Lin, L. et al. Gut https://doi.org/10.1136/gutjinl-2020-321013 (2020)

7. Henwood, A. F. J. Histotechnol. https://doi.org/10.1080/01478885. 2020.1734718 (2020).

8. Banegas, J. R. et al. N. Engl. J. Med. 382, 786 (2020).

The authors declare no competing interests.

\title{
'Test, re-test, re-test': using inaccurate tests to greatly increase the accuracy of COVID-19 \\ testing
}

To the Editor - Commenting recently on rapid point-of-care tests, US COVID-19 coordinator Deborah Birx said, "We are very quality-oriented. We don't want false positives."

"If they are incredibly accurate, we will work out the quickest way to release them. If they are not accurate, we will not release any of them." echoed UK Chief Medical Officer Chris Whitty ${ }^{2}$.

Given the need for testing ${ }^{3}$, the end goal is a quick, accurate and cheap test. With scientific innovation, we will, in time, attain this goal. But the best is becoming the enemy of the good. Meanwhile, avoidable infections are growing.

The 'gold standard' RT-PCR test for COVID-19 is highly accurate and reproducible, but is costly (US\$125 per test kit, and over $\$ 15,000$ to set up a processing lab) and slow (4-6 hours of processing time, and a turnaround of 2-4 days, including shipping) ${ }^{4}$.

At the other extreme, a Bangladeshi lab has reportedly developed a $\$ 3$ rapid test kit that gives a result in under 15 minutes $\left(\right.$ ref. $\left.{ }^{4}\right)$. But the accuracy of such point-of-care tests is questionable.

Smart tactics can help break this tradeoff between cost and quality.

First, consider two quick, cheap and inaccurate tests, each developed by a different lab, and based on detection of

\begin{tabular}{|c|c|c|c|}
\hline $\begin{array}{c}\text { Flipping independent } \\
\text { fair coins }\end{array}$ & $\begin{array}{c}\text { Probability } \\
\text { of event }\end{array}$ & $\begin{array}{c}\text { Testing a patient repeatedly with independent tests, } \\
\text { each with a 50\% false-negative rate }\end{array}$ & $\begin{array}{c}\text { Probability } \\
\text { of event }\end{array}$ \\
\hline Heads & False negative \\
\hline Heads, heads & False negative, false negative \\
\hline $\begin{array}{c}\text { Heads, heads, } \\
\text { heads }\end{array}$ & False negative, false negative, \\
false negative
\end{tabular}

Fig. 1 | Why re-testing increases testing accuracy.

a different antibody - or of the same antibody, but via a different method. Suppose each test has a false-negative rate of $30 \%$, and, for simplicity, zero false-positive results. What if both tests were administered to the same person? If the results of the two tests are independent, the chances of obtaining two false-negative results drops to $9 \%$ (and to less than 3\% if a third independent test with similar characteristics is administered). Figure 1 illustrates this logic, which also applies to false-positive results, for a test with a $50 \%$ false-negative rate. (Reports suggest that the tests being considered for large-scale procurement in the $\mathrm{UK}$ are in this range $\left.\mathrm{e}^{4,5}\right)$. As a comparison, since 2017, rapid influenza diagnostic tests cleared by the US Food and Drug Administration have been required to achieve false-negative rates and false-positive rates of below $20 \%$ and $5 \%$, respectively, compared with RT-PCR ${ }^{6}$.

Second, this recommendation to test and re-test can apply elsewhere too. Consider a test that displays the same false-negative and false-positive rates as the tests above - and is also unreproducible. If a patient is tested twice in succession with this test, the results could vary. Counterintuitively, this lack of reproducibility may be advantageous. 
Again, if the results of the two tests are independent, the likelihood of two false-negative results drops to $9 \%$.

The implication is clear: even an inaccurate test tells us something. Or, to misquote the World Health Organization: 'test, re-test, re-test'.

Use of this strategy would be made easier if there were a database - updated in real time - of point-of-care tests being generated by labs around the world. This database, which could be assembled by an international organization such as the World Health Organization, would list the lab and test name, the antibody that the test detects (e.g., IgG, IgN or both ${ }^{7}$ ), the detection method (e.g., lateral-flow immunoassay) and its accuracy and reproducibility, the turnaround time, the testing-kit cost and the sample-processing cost. With this information in hand, governments and international organizations could advise scientists on what combination of cheap tests would be optimal for specific nations.

Third, consider a quick and cheap test with a $30 \%$ false-positive rate, and for simplicity, zero false-negative results. First, one could test many people with this test, and then test the subset who test positive with a highly accurate test. This economizes on the use of scarce but accurate test kits while allowing much wider testing than would have been possible with the few accurate test kits available. In short: 'test, triage, re-test'.

Finally, smart tactics can enable cheaper testing with the expensive RT-PCR tests, if a sample obtained can 'fuel' multiple tests. Some German hospitals are doing 'block tests' using a sample pooled from ten employees, and then are testing individually only if there is a positive result ${ }^{8}$.

One can take this idea further, by applying principles from discrete optimization. If the test is positive, then one would test two blocks of five samples each, and then further test the arm that tests positive. This mimics 'branch and bound' algorithms for solving discrete optimization problems such as the famous 'traveling salesperson' problem ${ }^{9}$, which requires finding the cheapest route for delivering supplies to a fixed number of stores.

These simple examples are illustrative. Naturally, several factors would come into play in their implementation. For example, block testing would increase time to diagnosis and may be more useful for asymptomatic low-risk cases.

Finally, all inaccuracies are not equal. Right now, tests with a high false-positive rate are less problematic - since people are being advised to stay home anyway - than those with high false-negative rates. Furthermore, a false-positive result for SARS-CoV-2 is unlikely to initiate treatment with negative side effects, as would chemotherapy for misdiagnosed cancer.

The key point here is that creative use of currently available cheap and quick tests - even if they are inaccurate and unreproducible - can go a long way to reaching adequate levels of accuracy and precision, at least until the gold-standard tests can be developed. of Surgery \& Cancer, Imperial College London, London, UK. ${ }^{3}$ Department of Economics, University of Oxford, Oxford, UK.

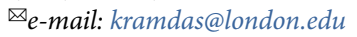

Published online: 12 May 2020

https://doi.org/10.1038/s41591-020-0891-7

\section{References}

1. Boseley, S. \& Kollewe, J. The Guardian https://www.theguardian. com/world/2020/mar/26/covid-19-self-test-could-allow-returnto-work-says-public-health-england (26 March 2020).

2. Boseley, S. The Guardian https://www.theguardian.com/world/ 2020/mar/25/uk-coronavirus-mass-home-testing-to-be-madeavailable-within-days (25 March 2020).

3. Ghebreyesus, T. A. \& Swaminathan, S. Lancet $\mathbf{3 9 5}$, 762-764 (2020).

4. Mahmud, F. Al Jazeera https://www.aljazeera.com/news/2020/ 03/bangladesh-scientists-create-3-kit-detect-covid-19200323035631025.html (24 March 2020)

5. Devlin, H. The Guardian https://www.theguardian.com/world/ 2020/apr/05/coronavirus-testing-kits-could-be-unreliableuk-scientists-say (5 April 2020).

6. Centers for Disease Control and Prevention. https://www.cdc. gov/flu/professionals/diagnosis/rapidlab.htm (4 February 2019).

7. Center for Health Security, Johns Hopkins School of Public Health. http://www.centerforhealthsecurity.org/resources/ COVID-19/200228-Serology-testing-COVID.pdf (28 February 2019).

8. Bennhold, K. The New York Times https://www.nytimes.com/ 2020/04/04/world/europe/germany-coronavirus-death-rate.html (4 April 2020).

9. Fisher, M. L. Manage. Sci. 27, 1-18 (1981).

\section{Acknowledgements}

We thank London Business School for providing Research and Materials Development funding in support of this research. Infrastructure support for this research was provided by the National Institute of Health Research Imperial Biomedical Research Centre.

\section{Competing interests}

A.D. has received a donation of one Axceed 260 machine from Bioscience Diagnostic Medical Technology in China, and is the academic sponsor on a study focusing on PCR and antibody testing for COVID-19 on behalf of the Department of Health.

\section{The fight to end tuberculosis must not be forgotten in the COVID-19 outbreak}

To the Editor - In the midst of the COVID19 pandemic, the world must remain vigilant to the potential for eruptions of tuberculosis (TB) and its drug-resistant (DR-TB) strains. Many countries with a high TB burden remain reliant on in-person and community-based directly observed therapy for TB treatment. With the current COVID19 conditions that affect mobility and access to care ${ }^{1-4}$, bothw seem impractical.

Global TB is exacerbated by the COVID19 pandemic. Regular treatment facilities are being closed because of a lack of resources, and TB could be misdiagnosed in settings in which COVID-19 testing is not available. Local governments must identify feasible options to retain patients with TB and DR-TB in care while fighting the COVID-19 pandemic. On 20 March 2020, the World Health Organization released an Information Note on TB and COVID-19 urging national TB programs to maintain continuity of essential services for people affected with TB during the COVID-19 pandemic ${ }^{5}$. It recommends providing adequate stocks of
TB medicines for all patients in order to ensure treatment completion without the patients' having to visit treatment centers unnecessarily to collect medications; this essentially forces the global TB program to shift from directly observed therapy to self-administered therapy. Digital-health technologies such as electronic medication monitors and video-supported therapy were also recommended to help patients adhere to their treatment. However, for optimal implementation of this strategy, trials evaluating the effectiveness of remote 